\title{
A Reduction Formula for Partitioned Matrices
}

\author{
Emilie V. Haynsworth
}

(April 13, 1960)

\begin{abstract}
A theorem of L. Goddard and H. Schneider, concerning square matrices $A$ and $B$, of orders $n$ and $m$, respectively, which satisfy an equation $A X=X B$ for some $n \times m$ matrix $X$, is generalized here for rectangular matrices $A$ and $B$, with dimensions $n_{1} \times n_{2}, m_{1} \times m_{2}$, which satisfy $A X_{2}=X_{1} B$, where $X_{i}$ has dimensions $n_{i} \times m_{i}$ for $i=1,2$. This result is used to find reduction formulas for partitioned matrices with submatrices, $A_{i j}$, having dimensions $n_{i} \times n_{j}$, and satisfying equations $A_{i j} X_{j}=X_{i} B_{i j}$. The reduction formulas given here are also generalizations of a theorem by $\mathrm{J}$. Williamson concerning partitioned matrices whose submatrices are all square and satisfy $A X=X B$, where $B$ is triangular and $X$ is square.
\end{abstract}

\section{Introduction}

Given a matrix $A=\left(a_{i j}\right)$ of order $n$, if there exists a nonsingular matrix $P$ such that

$$
\tilde{A}=P^{-1} A P=\left(\begin{array}{cc}
B & O \\
* & \vdots C
\end{array}\right)
$$

where $B$ is a square matrix of order $r$, and $O$ is an $r \times(n-r)$ matrix of zeros, $A$ is called a reducible matrix and the formula (1) is a reduction formula. The characteristic equation of $A$ is then factorable:

$$
\left|A-\lambda I_{n}\right|=\left|B-\lambda I_{r}\right|\left|C-\lambda I_{n-r}\right|
$$

where $I_{n}$ represents the identity matrix of order $n$.

The results in this paper concern a reduction formula of the type (1) for partitioned matrices, and give a connection between, and an extension of, results by L. Goddard and H. Schneider, ${ }^{1}$ and J. Williamson. ${ }^{2}$ The theorem of Goddard and Schneider is generalized in theorem 1, and this is applied in theorem 2 to give a general reduction formula for partitioned matrices,

$$
A=\left(\begin{array}{cccc}
A_{11} & A_{12} & \ldots & A_{1 t} \\
A_{21} & A_{22} & \ldots & A_{2 t} \\
& & & \\
A_{t 1} & A_{t 2} & \ldots & A_{t t}
\end{array}\right)
$$

where the submatrices $A_{i j}$ have dimensions $n_{i} \times n_{j}$, $i, j=1, \ldots, t$.

Theorem 2 contains the results of Goddard and Schneider and those of Williamson as special cases.

${ }^{1}$ L. S. Goddard and H. Schneider, Matrices with a nonzero commutator, Proc. Camb. Phil. Soc. 51, 551 (1955).

${ }^{2} \mathrm{~J}$. Williamson, The latent roots of a matrix of special type, Bul. Am. Math. Soc. 37,585 (1931),

\section{Results of Goddard and Schneider}

Goddard and Schneider showed that if two square matrices $A$ and $B$ of orders $n$ and $m$, respectively, are related by the equation,

$$
A X=X B
$$

where $X$ is an $n \times m$ matrix of rank $r$, then there exist matrices $P$ and $Q$, depending on $X$, such that

$$
P^{-1} A P=\left(\begin{array}{ll}
E & * \\
O & C
\end{array}\right), \quad Q^{-1} B Q=\left(\begin{array}{cc}
E & O \\
* & D
\end{array}\right),
$$

where $E$ is square, of order $r$, so that $A$ and $B$ have $r$ roots in common.

More generally, Goddard and Schneider proved, for any $m \times n$ matrix $K$ and any polynomial $f(x, y)$,

$$
\begin{aligned}
& P^{-1} f(A, X K) P=\left(\begin{array}{cc}
f(E, G) & * \\
O & f(C, 0)
\end{array}\right) \\
& Q^{-1} f(B, K X) Q=\left(\begin{array}{cc}
f(E, G) & O \\
* & f(D, 0)
\end{array}\right)
\end{aligned}
$$

where $\mathrm{G}$ depends on $K$. So all such functions of $A$ and $B$ are reducible if $r<\min (m, n)$, and each corresponding pair (for which $f$ and $K$ are the same) has $r$ roots in common.

If $r=m<n, \quad A$ and $f(A, X K)$ are reducible and (1) holds for $A$ and $B$ as defined in (3) and $C$ as defined in (4).

If $r=m=n$, for the matrices in (3), we have $X^{-1} A X=B$, so if $B$ is reduced, $A$ and $f(A, X K)$ are reducible. Naturally, similar results would hold for $B$ if $r=n \leqq m$.

Thus, although the results of Goddard and Schneider concern a pair of matrices, the cases given above can be considered as leading to a reduction formula for one or both of the matrices. 


\section{Results of Williamson}

J. Williamson ${ }^{2}$ deals with partitioned matrices as in (2) in which all submatrices, or blocks, are square. He showed that if a partitioned matrix $A$, of order $n t$, has blocks of order $n$ which can be simultaneously reduced to triangular form, then $A$ is reducible to a block-triangular matrix, i.e., a partitioned matrix (2) in which $A_{i j}=0, \quad j>i$.

Williamson's results could be expressed as follows:

Given a partitioned matrix $A=\left(A_{i j}\right)$ of order $n t$, with blocks of order $n$, if there exists a nonsingular, $n \times n$ matrix $X$ such that

$$
X^{-1} A_{i j} X=B_{i j}
$$

or

$$
A_{i j} X=X B_{i j}, \quad(i, j=1, \ldots, t)
$$

where $B_{i j}$ is triangular with elements $\lambda^{(i j)}, \ldots ., n^{(i j)}$ on the diagonal, then $A$ is similar to a block-triangular matrix with blocks

$$
\tilde{A}_{k k}=\left(\lambda_{k}^{(i j)}\right)
$$

on the diagonal.

This theorem is then generalized by Williamson to show that, given any partitioned matrix $A=\left(A_{i j}\right)$ satisfying (6) with $B_{i j}$ triangular, the partitioned matrix which has blocks

$$
G_{i j}=f_{i j}\left(A_{i j}\right)
$$

where $f_{i j}(A)$ is a rational function of $A$ with nonsingular denominator, has as roots the roots of the $n$ matrices of order $t$,

$$
\tilde{G}_{k k}=f_{i j}\left(\lambda_{k}^{(i j)} \quad(k=1, \ldots, n ; i, j=1, \ldots, t) .\right.
$$

\section{Connection Between Theorems of Goddard and Schneider and of Williamson}

It can be seen, from eqs (3) and (6), that although the theorem by Williamson and that of Goddard and Schneider are quite different, there is a connection between them.

In theorem 1 we generalize the results of Goddard and Schneider to rectangular matrices $A$ and $B$ of dimensions $n_{1} \times n_{2}$ and $m_{1} \times m_{2}$, respectively, satisfying

$$
A X_{2}=X_{1} B
$$

where $X_{i}$ is $n_{i} \times m_{i}$ of $\operatorname{rank} r_{i}, \quad i=1$ or 2 .

We then apply this result in theorem 2 to square partitioned matrices $A$ and $B$, with rectangular blocks $n_{i} \times n_{j}$ and $m_{i} \times m_{j}$, respectively, in which the following relations hold between the blocks,

$$
A_{i j} X_{j}=X_{i} B_{i j}, \quad(i, j=1, \ldots, t)
$$

where $X_{i}$ is $n_{i} \times m_{i}$ of rank $r_{i}$. ${ }^{2} \mathrm{~J}$. Williamson The latent roots of a matrix of special type, Bul. Am. Math.
Then the theorem of Goddard and Schneider can be obtained as a special case of theorem 2 when we set $t=1$ in (8). If we have $r_{i}=m_{i}=n_{i}=n, i=1$, $\ldots, t$, and $B_{i j}$ triangular, $i, j=1, \ldots, t$, we have the theorem of Williamson.

\section{Theorem of Goddard and Schneider for Rectangular Matrices}

The proof of theorem 1 is essentially the same as that of Goddard and Schneider, the only difference being the addition of subscripts to correspond with the two different matrices, $X_{1}$ and $X_{2}$.

Theorem 1. If $A$ and $B$ are rectangular matrices satisfying ( $(7)$, there exist matrices $P_{1}, \dot{P}_{2}, Q_{1}$ and $Q_{2}$, such that

$$
\begin{aligned}
& P_{1}^{-1} A P_{2}=\left(\begin{array}{ll}
E & * \\
O & C
\end{array}\right), \\
& Q_{1}^{-1} B Q_{2}=\left(\begin{array}{ll}
E & O \\
* & D
\end{array}\right),
\end{aligned}
$$

where $E$ is an $r_{1} \times r_{2}$ matrix, and $C$ and $D$ are rectangular matrices having dimensions $\left(n_{1}-r_{1}\right) \times\left(n_{2}-r_{2}\right)$ and $\left(m_{1}-r_{1}\right) \times\left(m_{2}-r_{2}\right)$, respectively. Moreover, if $f(x, y)$ is a polynomial in two noncommutative indeterminates (which is linear and homogeneous if $A$ and $B$ are not square, or if $r_{1} \neq r_{2}$ ) and $K$ is an arbitrary $m_{1} \times$ $n_{2}$ matrix,

$$
\begin{aligned}
& P_{1}^{-1} f\left(A, X_{1} K\right) P_{2}=\left(\begin{array}{cc}
f(E, G) & * \\
0 & f(C, 0)
\end{array}\right), \\
& Q_{1}^{-1} f\left(B, K X_{2}\right) Q_{2}=\left(\begin{array}{cc}
f(E, G) & 0 \\
* & f(D, 0)
\end{array}\right),
\end{aligned}
$$

where $G$ depends upon $K$.

Proof. For $i=1$ or 2 , we have $X_{i}$ of rank $r_{i}$, so there exist corresponding nonsingular matrices, $P_{i}$ and $Q_{i}$, of order $n_{i}$ and $m_{i}$, respectively, such that

$$
Y_{i}=P_{i}^{-1} X_{i} Q_{i}=\left(\begin{array}{cc}
I_{r_{i}} & 0 \\
0 & 0
\end{array}\right)
$$

is an $n_{i} \times m_{i}$ matrix with an identity matrix of order $r_{i}$ in the upper left corner.

$$
\begin{aligned}
& \text { Let } \\
& \tilde{A}=P_{1}^{-1} A P_{2}, \quad \tilde{B}=Q_{1}^{-1} B Q_{2}, \quad \tilde{K}=Q_{1}^{-1} K P_{2}
\end{aligned}
$$

and partition each matrix so that there is an $r_{1} \times r_{2}$ matrix in the upper left corner, i.e.,

$$
\tilde{A}=\left(\begin{array}{cc}
\tilde{A}_{11} & \tilde{A}_{12} \\
\tilde{A}_{21} & \tilde{A}_{22}
\end{array}\right),
$$


and $B$ and $K$ are partitioned similarly.

Then, from (11) and (12),

$$
\begin{aligned}
& P_{1}^{-1} A X_{2} Q_{2}=\tilde{A} Y_{2}=\left(\begin{array}{cc}
\tilde{A}_{11} & 0 \\
\tilde{A}_{21} & 0
\end{array}\right), \\
& P_{1}^{-1} X_{1} B Q_{2}=Y_{1} \tilde{B}=\left(\begin{array}{cc}
\tilde{B}_{11} & \tilde{B}_{12} \\
0 & 0
\end{array}\right) .
\end{aligned}
$$

Since, by (7), $\tilde{A} Y_{2}=Y_{1} \tilde{B}$, (13) implies

$$
\tilde{A}_{11}=\widetilde{B}_{11}, \quad \tilde{A}_{21}=\tilde{B}_{12}=0 .
$$

Thus, if we let $\tilde{A}_{11}=E, \quad \tilde{A}_{22}=C, \quad \tilde{B}_{2 \dot{ }}=D$, from (12) and (14) we have (9). Also

$$
\begin{gathered}
P_{1}^{-1} X_{1} K P_{2}=Y_{1} \tilde{K}=\left(\begin{array}{cc}
\tilde{K}_{11} & \tilde{K}_{12} \\
0 & 0
\end{array}\right) \\
Q_{1}^{-1}\left(K X_{2}\right) Q_{2}=\tilde{K} Y_{2}=\left(\begin{array}{cc}
\tilde{K}_{11} & 0 \\
\tilde{K}_{21} & 0
\end{array}\right)
\end{gathered}
$$

so if we let $\tilde{K}_{11}=G$, we obtain (10).

\section{A General Reduction Formula}

In this section we apply theorem 1 to partitioned matrices with rectangular blocks satisfying (8). We will in general obtain formulas of the type (4) which can be considered as reduction formulas for $A$ or $B$ or both.

Specific applications of this theorem in the reduction of certain special partitioned matrices have been given previously by the author. ${ }^{3}$

Before applying theorem 1 to theorem 2 it will be necessary to prove the following.

Lemma. Given a partitioned matrix of order $N$, with $n_{i} \times n_{j}$ blocks $A_{i j}$, if

$$
A_{i j}=\left(\begin{array}{cc}
B_{i j} & 0 \\
* & C_{i j}
\end{array}\right)
$$

where all matrices $B_{i j}$ are square, of order $r$, and the matrices $C_{i j}$ are $\left(n_{i}-r\right) \times\left(n_{j}-r\right)$, then $A$ is reducible as in (1) where $P$ is a permutation matrix, and $B=\left(B_{i j}\right), \quad C=\left(C_{i j}\right)$.

Proof. The proof consists merely in defining the permutation matrix $P$, which is equivalent to writing the order in which the rows and columns of $A$ should be arranged.

${ }^{3}$ E. Haynsworth, Applications of a theorem on partitioned matrices, J. Research NBS 63B, 73 (1959).
Let $\sum_{i=1}^{k} n_{i}=N_{k}$. Then $N_{1}=n_{1}, \quad N_{t}=N$. If we arrange the rows and columns of $A$ in the following order:

$$
\begin{array}{cccc}
1, & 2, & \ldots, & r ; \\
N_{1}+1, & N_{1}+2, & \ldots & N_{1}+r ; \\
& \ldots & & \\
N_{t-1}+1, & N_{t-1}+2, & \ldots, & N_{t-1}+r ; \\
r+1, & r+2, & \ldots, & N_{1} ; \\
N_{1}+r+1, & N_{1}+r+2, \ldots, & N_{2} ;
\end{array}
$$

$$
N_{t-1}+r+1, N_{t-1}+r+2, \ldots, \quad N_{t} \text {; }
$$

we have a new matrix $A$ in which the matrices $B_{i j}$ are together in the upper left corner, and the matrices $C_{i j}$ are together in the lower right corner, so $\tilde{A}$ will have the form (1).

Theorem 2. Suppose we have partitioned matrices, $A$ and $B$, with rectangular blocks, $A_{i j}$ and $B_{i j}$, satisfying (8), where $r_{i}=r, \quad i=1, \ldots, t$. Then, $A$ and $B$ have tr roots in common.

Moveover, if $G=\left(G_{i j}\right)$ and $H=\left(H_{i j}\right)$ have rectangular blocks,

$$
G_{i j}=f_{i j}\left(A_{i j}, X_{i} K_{i j}\right), \quad H_{i j}=f_{i j}\left(B_{i j}, K_{i j} X_{j}\right)
$$

where the matrices $K_{i j}$ are arbitrary $m_{i} \times n_{j}$ matrices and the polynomials $f_{i j}(x, y)$ are as defined in theorem 1, then all pairs of matrices defined in (18) have tr roots in common.

Proof. Let $P_{i}$ and $Q_{i}$ be matrices satisfying (11) for $i, j=1, \ldots, t$ and let $P$ and $Q$ be the direct sums of the matrices $P_{i}$ and $Q_{i}$ respectively, i.e.,

$$
P=P_{1}+P_{2} \dot{+} \cdots+P_{t}=\sum_{i=1}^{t} \cdot P_{i}, Q=\sum_{i=1}^{t} \cdot Q_{i} .
$$

Then we have, using block multiplication of matrices,

$$
P^{-1} A P=\tilde{A}=\left(\tilde{A}_{i j}\right), \quad Q^{-1} B Q=\tilde{B}=\left(\tilde{B}_{i j}\right),
$$

where

$$
\tilde{A}_{i j}=P_{i}^{-1} A_{i j} P_{j}, \quad \tilde{B}_{i j}=Q_{i}^{-1} B_{i j} Q_{j} .
$$

Thus, from (9),

$$
\tilde{A}_{i,}=\left(\begin{array}{ll}
E_{i j} & * \\
O & C_{i j}
\end{array}\right), \quad \tilde{B}_{i j}=\left(\begin{array}{ll}
E_{i j} & 0 \\
* & D_{i j}
\end{array}\right) .
$$


So, by the lemma, $\tilde{A}$ and $\tilde{B}$ are similar to the matrices $\hat{A}$ and $\hat{B}$,

$$
\hat{A}=\left(\begin{array}{ll}
E & * \\
O & C
\end{array}\right), \quad \hat{B}=\left(\begin{array}{cc}
E & O \\
* & D
\end{array}\right),
$$

where $E=\left(E_{i j}\right), \quad C=\left(C_{i j}\right), \quad D=\left(D_{i j}\right)$. Thus the roots of $E$ are roots of both matrices.

Also, using (15) for the matrices $K_{i j}, P_{i}, P_{j}, Q_{i}, Q_{j}$, we can in the same way obtain the more general result concerning the matrices $G$ and $H$.

Corollary 1. If a matrix $A$ of order nt can be partitioned into square blocks $A_{i j}$, of order $n$, having $r$ linearly independent characteristic vectors $x_{h}$ in common, corresponding to the roots, $\lambda_{h}^{(i j)}, h=1, \ldots, r$; $i, j=1, \ldots, t$, then tr of the roots of $A$ are roots of the matrices $\left(\lambda_{h}^{(i j)}\right)$.

Proof: Let

$$
X_{i}=X=\left(x_{1}, x_{2}, \ldots, x_{r}\right)
$$

be the $n \times r$ matrix whose columns are the given vectors and

$$
B_{i j}=\operatorname{diag}\left(\lambda_{1}^{(i j)}, \lambda_{2}^{(i j)}, \ldots, \lambda_{r}^{(i j)}\right) .
$$

Then (8) holds for the blocks $A_{i j}$, and $\operatorname{tr}$ of the roots of $A$ are roots of $B=\left(B_{i j}\right)$. If we now rearrange the rows and columns of $B$ in the order,

$$
\begin{aligned}
& 1, \quad r+1, \quad 2 r+1, \ldots,(t-1) r+1 ; \\
& 2, \quad r+2, \quad 2 r+2, \ldots,(t-1) r+2 \text {; } \\
& r, 2 r, \ldots, t r
\end{aligned}
$$

$B$ will be in block-diagonal form with the matrices $\left(\lambda_{h}^{(i j)}\right)$ on the diagonal.

This corollary is applied by the author to certain special partitioned matrices (see footnote 3 ).

Corollary 2. If a matrix $A$, of order nt, can be partitioned into square submatrices of order $n$ which are mutually commutative and have roots, $\lambda_{h}^{(i i)}, \quad(h=1$, . . . , $n)$, the roots of $A$ are the roots of the $n$ matrices, $\left(\lambda_{1}^{(i j)}\right),\left(\lambda_{2}^{(i j)}\right), \ldots .,\left(\lambda_{n}^{(i j)}\right)$.

This corollary would follow also from Williamson's theorem since any set of mutually commutative matrices can be simultaneously triangularized.

The author is grateful for the helpful suggestions of Dr. A. Ostrowski and Prof. H. Schneider.

(Paper 64B3-33) 